Trauma Berufskrankh 2016 · 18 (Suppl 5):S479-S485 DOI 10.1007/s10039-016-0175-0

Online publiziert: 21. Juli 2016

(c) Springer-Verlag Berlin Heidelberg 2016

CrossMark

M. Hoffmann $\cdot$ M. Runge $\cdot$ P. Dufek $\cdot$ K.-C. Westphal

Klinik für Orthopädie und Unfallchirurgie, Schön Klinik Neustadt, Neustadt in Holstein, Deutschland

\title{
Posttraumatische Korrekturen und Revision am proximalen Femur
}

Proximale Femurfrakturen zählen zu den häufigsten Frakturen am menschlichen Skelett über alle Altersklassen hinweg [3]. Die operative Versorgung dieser Frakturen ist bisweilen anspruchsvoll und führt bei inadäquater Reposition und Fixation zu suboptimalen Ergebnissen, die im Verlauf posttraumatische Korrekturen und Revisionen erforderlich machen [3]. Die Planung der Revisionsoperation erfordert eine umfassende, multifaktorielle Fehleranalyse unter Berücksichtigung zahlreicher Parameter. Dazu gehören neben der Ergründung des Osteosyntheseversagens die Lokalisation der Fraktur sowie deren exakte Klassifizierung, das Patientenalter, der Knochenstatus und vieles mehr. Zudem sollte der Grund des Osteosyntheseversagens evaluiert werden. Falsche Indikationsstellung, operationstaktische Fehler und die Wahl falscher Implantate beeinflussen maßgeblich die Knochenheilung und damit das Rennen zwischen Knochenheilung und Implantatversagen, das bei ausbleibender Knochenheilung regelhaft auftritt.

Am proximalen Femur muss zwischen verschiedenen Frakturentitäten und Subgruppen unterschieden werden [9]. Die häufigsten Subgruppen mit Korrekturbedarf stellen neben den medialen Schenkelhalsfrakturen die extraartikulären proximalen Femurfrakturen dar, die in per-, inter- und subtrochantäre Frakturen subklassifiziert werden [9].

\section{Mediale Schenkelhalsfrakturen}

Bis auf wenige Ausnahmen werden mediale Schenkelhalsfrakturen regelhaft operativ versorgt $[8,11]$. Während bei älteren Patienten oder vorbestehenden koxarthrotischen Veränderungen der endoprothetische Gelenkersatz zum Einsatz kommt [8], wird beim jungen Patienten die Fraktur osteosynthetisch versorgt, da die Rekonstruktion mit steigendem Alter zu einem schlechteren funktionellen Outcome führt [11]. Zur osteosynthetischen Versorgung stehen unterschiedliche Implantatsysteme zur Verfügung. Neben der 3-Schrauben-Osteosynthese, der dynamischen Hüftschraube mit und ohne Antirotationsschraube und der Marknagelosteosynthese stehen seit Kurzem auch Kombinationssysteme aus Schrauben und winkelstabilen Platten zur Verfügung (• Abb. 1). In aktuellen Studien [6] zeigen diese Kombinationssysteme aus winkelstabiler Plattenosteosynthese und Schenkelhalsschrauben vielversprechende biomechanische Ergebnisse. Auch in ersten klinischen Studien zeigen diese Implantate signifikant reduzierte $\mathrm{Re}$ visionsraten aufgrund mechanischer Komplikationen. Die Rate an avaskulären Hüftkopfnekrosen bleibt jedoch davon unbeeinflusst [13].

Trotz verbesserter mechanischer Eigenschaften moderner unfallchirurgischer Implantate kommt es bei ausbleibender Frakturheilung oder avaskulärer Hüftkopfnekrose zum Versagen der Osteosynthese und $\mathrm{zu}$ einer Revisionsbedürftigkeit [13]. Die Indikationsstellung zur Revision und das entsprechende Verfahren sollten anhand verschiedener Parameter, unter anderem dem Patientenalter, dem Knochenstatus und der Versagensanalyse des osteosynthetischen Implantats, festgelegt werden. Das Revisionsspektrum beinhaltet da- bei im Wesentlichen 2 Bereiche: Zum Erhalt bzw. Rekonstruktionsversuch der Biologie im Sinne einer hüftkopferhaltenden Revisionsoperation stehen diverse Reosteosyntheseverfahren zur Verfügung. Sollte eine Reosteosynthese nicht möglich sein, steht der gelenkendoprothetische Ersatz zur Verfügung.

\section{Revision im Sinne einer Reos- teosynthese bei Pseudarthrosen im Bereich des medialen Schenkelhalses}

Je jünger der Patient, je technisch inadäquater der Voreingriff, je besser die Kopfqualität und je kopfferner das Versagen ist, desto besser ist die Erfolgsrate mittels Reosteosynthese. Standardmethode der Reosteosynthese ist die Umstellungsosteosynthese nach Pauwels [4]. Mittels proximaler valgisierender intertrochantärer Osteotomie werden Scherkräfte in Kompressionskräfte verwandelt, die in der Folge zu einer Frakturheilung führen. Die Effektivität dieser Behandlungsmethode konnte in zahlreichen Studien bestätigt werden [4].

Um die Gefahr des Implantatversagens bei medialen Schenkelhalsfrakturen zu minimieren, sollte die genaue Indikationsstellung anhand zuverlässiger Klassifikationssysteme erarbeitet werden. Die Garden-Klassifikation bietet nur ein koronares Frakturklassifikationssystem. Aus aktuellen Studien ist jedoch bekannt, dass gerade Frakturen mit posterolateraler Trümmerzone im Bereich des Schenkelhalses häufiger zu einem sekundären Repositionsverlust und Implantatversagen führen [10]. Gemäß der aktuellen Literatur sind als Garden I und 

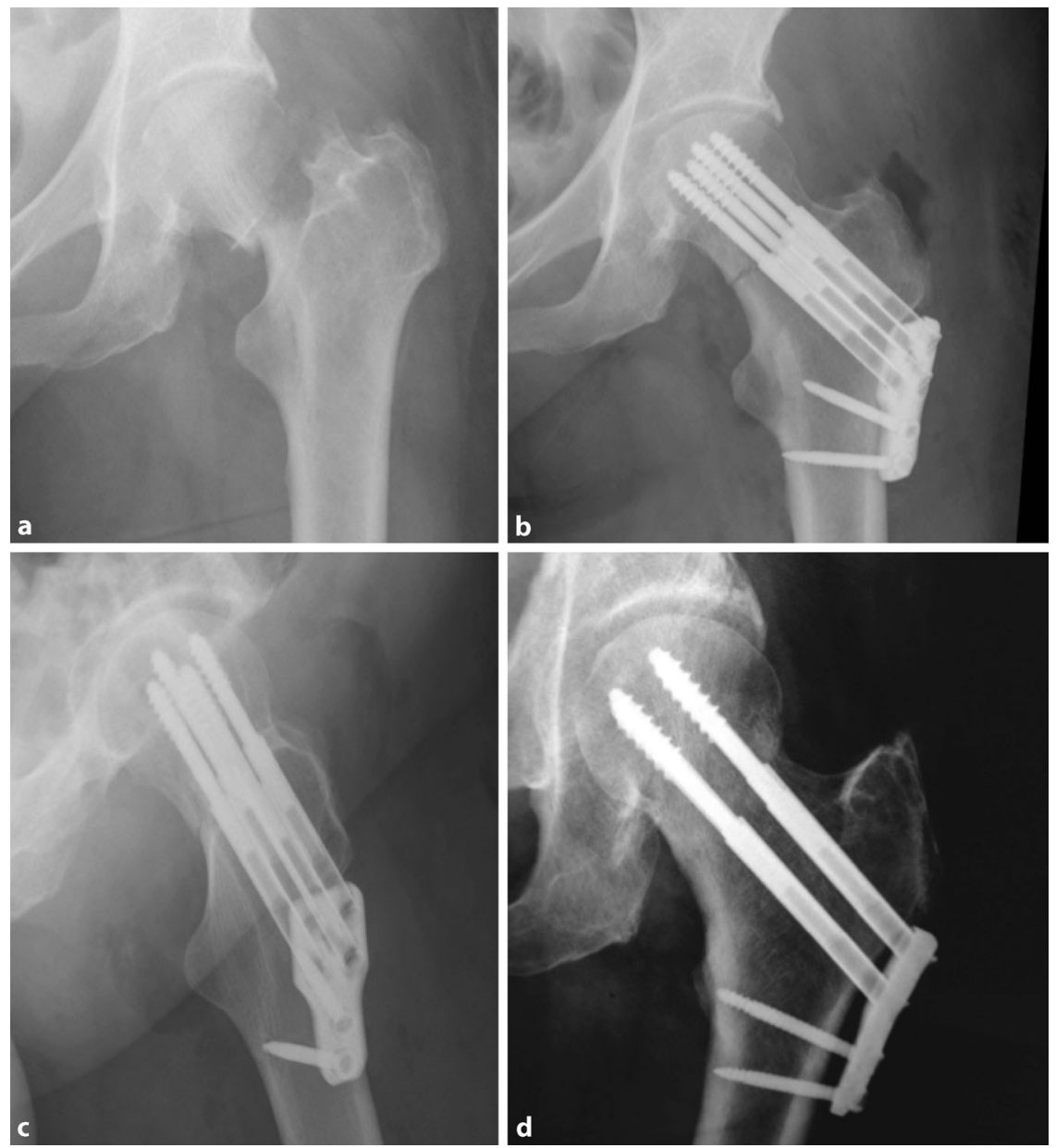

Abb. $1 \Delta$ a Dislozierte mediale Schenkelhalsfraktur mit posterolateraler Trümmerzone (Garden Typ IV). b, c Versorgung mittels Targnon FN, Fa. Aesculap. d Knöcherne Konsolidierung der Fraktur ohne wesentliche Offset-Reduktion

Garden II klassifizierte Schenkelhalsfrakturen kopferhaltend mittels Osteosynthese versorgbar, während Garden-Typ-IIIund -IV-Frakturen mit einem hohen osteosynthetischen Versagensrisiko und einer hohen Rate an avaskulären Hüftkopfnekrosen einhergehen [10].

Trotz der bereits angesprochenen biomechanisch verbesserten Implantatsysteme konnte zwar die biomechanische Fehlerrate signifikant reduziert werden. Dennoch: Die Rate an Osteonekrosen bleibt von den verbesserten biomechanischen Eigenschaften in der Regel jedoch unbeeinflusst [3].

\section{Implantation einer Hüftgelenk- endoprothese}

Ist im Rahmen einer posttraumatischen Korrektur nicht mehr von einer erfolgreichen Wiederherstellung des Kopf-Hals- hüftgelenkendoprothesen eignen sich für die Revision.

Im Rahmen des Osteosyntheseversagens bei medialen Schenkelhalsfrakturen kann es zu einer deutlichen Impression des Kopf-Hals-Fragments kommen, die wiederum $\mathrm{zu}$ einem Offset-Verlust am Hüftgelenk führt. Aus biomechanischen Studien ist bekannt, dass eine Offset-Reduktion konsekutiv aufgrund des verminderten Hebelarmes zu einer Insuffizienz der Glutealmuskulatur, insbesondere des Glutaeus medius führt [2]. Dieser Offset-Verlust geht häufig neben dem Osteosyntheseversagen mit einem typischen Trendelenburg-Hinken einher. Die Wiederherstellung der biomechanischen Parameter muss vor einer Revision gründlich geplant werden. Aufgrund der chronischen Reduktion des Offsets durch sekundäre Fraktursinterung kann es zu Problemen bei der Wiederherstellung kommen. Dies muss entsprechend antizipiert werden.

\section{Proximale, extraartikuläre Femurfrakturen}

Im Bereich der proximalen Femurfrakturen existieren im Wesentlichen 3 Entitäten:

- die pertrochantären,

- die intertrochantären und

- die subtrochantären Femurfrakturen [9].

Fragments auszugehen, ist eine gelenkersetzende endoprothetische Versorgung anzustreben. In zahlreichen Studien konnte gezeigt werden, dass die Ergebnisqualität einer Revisionsendoprothese nach Osteosyntheseversagen bei medialer Schenkelhalsfraktur zuverlässig gute Ergebnisse zeigt [1]. Dennoch ragen die funktionellen Ergebnisse der Revisionsendoprothetik nach fehlgeschlagener Osteosynthese nicht an die der primären Endoprothetik in der Fraktursituation heran und verfehlen die Ergebnisqualität des endoprothetischen Gelenkersatzes bei Koxarthrose um ein Weites. So werden in der Literatur unterschiedlich hohe Komplikationsraten im Sinne von erhöhten periprothetischen Frakturrisiken sowie erhöhten Luxationsraten berichtet [1]. Sowohl zementierte als auch nicht zementierte oder Hybrid-

\section{Prävention einer notwendigen Korrektursituation}

Die Prävention posttraumatischer Korrekturen lässt sich im Wesentlichen durch folgende Faktoren beeinflussen: anatomische Reposition, Minimierung des TipTo-Apex-Abstandes (TAD), korrekte Definition des Eintrittspunktes, die zwingende anatomische Reposition vor Beginn der Marknagelosteosynthese, Kompression der Fraktur sowie Wahl des geeigneten Implantates und korrekte Indikationsstellung für Cerclagen. Im Vergleich der per-, inter- und subtrochantären Femurfrakturen zeigt die Nagelosteosynthese biomechanische Vorteile gegenüber den extramedullären Implantaten [9]. Aufgrund des intramedullären Kraftträgers verringert sich der bio- 
mechanische Hebelarm bis in die Spitze des Femurkopfes. Aufgrund dieser Eigenschaft scheinen intramedulläre Kraftträger den extramedullären, lateralen Fixationssystemen (z. B. Dynamische Hüftschraube [DHS], Winkelplatten) überlegen $\mathrm{zu}$ sein. Insbesondere im Bereich von Frakturen, die die laterale Begrenzung des Trochanter-major-Massivs tangieren, bieten intramedulläre Kraftträger eine Abstützung nach lateral für das mediale Kopf-Hals-Fragment. Zudem können Marknagelosteosynthesen über minimalinvasive Zugänge implantiert werden: In der Fraktursituation ist das proximal der Fraktur lokalisierte Femur in der Regel in Flexion und Abduktion disloziert.

Über minimalinvasive Verfahren kann mittels Kugelspieß vor Implantation des Marknagels eine anatomische Reposition herbeigeführt werden, die mit dem Marknagel dann anatomisch retiniert werden muss (-Abb. 2). Wichtig für eine zeitgerechte Frakturheilung sind die Minimierung des TAD sowie die Ausrichtung der Schenkelhalsschraube in eine Center-Center-Position im Kopfbereich [12]. Insbesondere die Minimierung des TAD zeigt in der Literatur eine signifikante Herabsetzung des Cutouts mit sekundärem Materialversagen ([12]; - Abb. 3). Ist eine intraoperative Kompression der Fraktur über das Implantat möglich, so ist eine statische Verriegelung indiziert. Mit der statischen Verriegelung wird die Gefahr von sekundären Impaktionen im Bereich des Schenkelhalses respektive der Fraktur minimiert. Damit wird ebenfalls das Risiko eines Offset-Verlusts und der damit assoziierten Beeinflussung des Glutaeusmedius-Hebelarms reduziert.

\section{Timing bei notwendiger Revisionsoperation}

Bei notwendiger Revisionsoperation kommt dem Timing eine entscheidende Bedeutung zu. Dabei wird eine frühzeitige (unmittelbar intraoperative oder frühzeitig postoperative) Revision von einer Revision im Verlauf unterschieden. Möglichst frühzeitige, intraoperative Korrekturen und Revisionen sind anzustreben, erfordern aber eine kri-

Trauma Berufskrankh 2016 · 18 (Suppl 5):S479-S485 DOI 10.1007/s10039-016-0175-0

(c) Springer-Verlag Berlin Heidelberg 2016

M. Hoffmann · M. Runge $\cdot$ P. Dufek · K.-C. Westphal

Posttraumatische Korrekturen und Revision am proximalen Femur

\section{Zusammenfassung}

Proximale Femurfrakturen zählen zu den häufigsten Frakturen des menschlichen Skeletts über alle Altersklassen hinweg. Fehlgeschlagene Osteosynthese erfordert posttraumatische Korrekturen. Da die Ergebnisqualität von Revisionsoperationen in der Regel schlechter als die Ergebnisqualität bei adäquat stattfindender Frakturheilung ist, kommt der Prävention eines Osteosyntheseversagens durch die Wahl des richtigen Implantats und der richtigen Technik eine entscheidende Bedeutung zu. Die frühzeitige Identifikation möglicher komplikationsträchtiger Verläufe ist von entscheidender Bedeutung. Insbesondere bei jungen Patienten steht ein biologischer
Rekonstruktionsversuch im Vordergrund. Dieser ist in der Regel erfolgreicher, je technisch inadäquater der Voreingriff war, je besser das proximale Kopffragment und die Gelenkqualität sind und je kopfferner das Versagen der Osteosynthese lokalisiert ist. Bei älteren Patienten ist in der Regel ein endoprothetischer Ersatz notwendig. Bei allen Revisionen ist an mögliche Infektionen zu denken und das Vorliegen dieser mittels Punktion oder offener Biopsie zu sichern oder auszuschließen.

Schlüsselwörter

Prothese - Implantat - Osteosynthese . Infektion · Biopsie

\section{Posttraumatic corrections and revision of the proximal femur}

\section{Abstract}

Proximal femoral fractures represent one of the most common fracture entities in trauma surgery. Failed osteosyntheses require revision surgery and posttraumatic corrections. As revision surgery results are inferior compared to primary bone healing, failure prevention by choosing the correct implant and technique is mandatory as is the early identification of complications. Especially in young patients, preservation and biological restoration with consecutive bone healing is the treatment of choice. In young patients, reconstructive surgery is likely to be successful, the more inadequate the previous surgery was, the better the head-neck fragment quality is, and the more distal the failure is located in relation to the head-neck fragment. In terms of revision surgery, elderly patients usually require total hip arthroplasty. Infections must be either diagnosed or excluded by joint aspiration or open biopsy.

\section{Keywords}

Prosthesis - Implants · Osteosynthesis . Infection · Biopsy tische Analyse der Osteosynthese und frühestmögliche Identifikation von Fehlerquellen.

\section{Infektionen}

Bei ausbleibender Frakturheilung, regelrechter Implantation und sonst unauffälligem Verlauf sollte stets nach Ausschluss anderer Möglichkeiten an eine Infektion gedacht werden. Ungewöhnliche periartikuläre Verkalkungen können das Vorliegen einer periimplantären Infektion bereits in der Frühphase anzeigen. Zur Sicherung einer möglichen vorliegenden Infektion sind eine Punktion, ggf. eine offene Biopsie sowie die Evaluation der hämatologischen Parameter indiziert. Im Bereich der Frakturversorgung werden unterschiedliche periimplantäre Infektionen unterschieden [14]. Neben Frühinfektion (weniger als 2 Wochen) kann es zu Spätinfektionen (2 bis 10 Wochen) sowie zu Low-grade-Infektionen (>10 Wochen) kommen. Die Infektionsmodi sind in der Regel mit modusspezifischen Keimen assoziiert. Dabei spielen insbesondere im Bereich von periimplantären Frühinfekten Staphylococcus-aureus-Infekte mit einer Frequenz von um die $30 \%$ eine entscheidende Rolle [14]. Die Sicherung eines möglichen vorliegenden Infekts ist von entscheidender Bedeutung, da dies die Revisionsoptionen beeinflusst. Grundsätzlich ist $\mathrm{zu}$ entscheiden, ob das Implantat erhalten, 

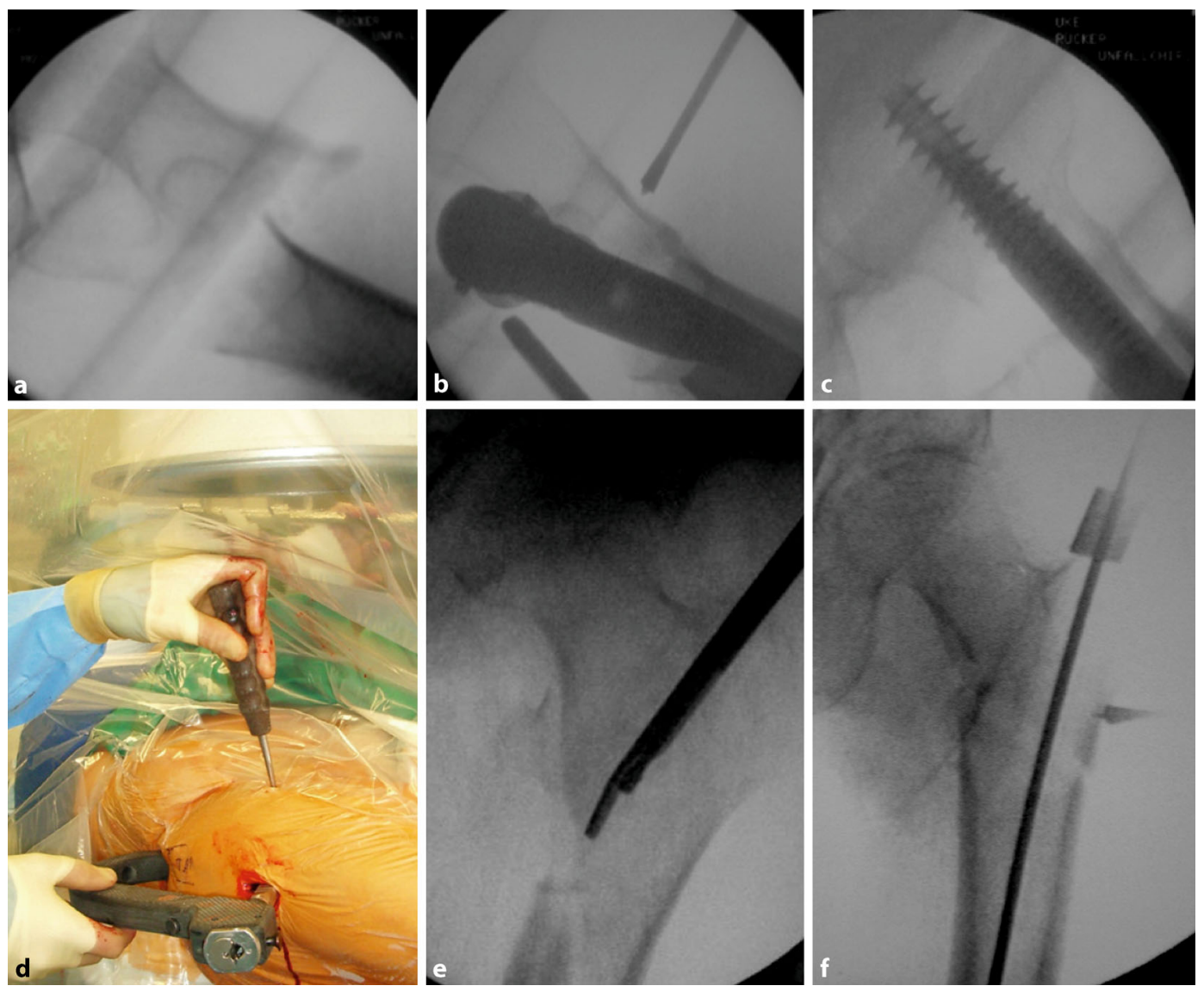

Abb. $2 \triangleleft$ a In Flexion disloziertes Kopf-Hals-Fragment. b Minimalinvasive Reposition über einen Kugelspieß und fluoroskopische Kontrolle. d, e Frakturreposition über eine Repositionshilfe. f Neutralisierung der Abduktionsfehlstellung über einen Kugelspieß von lateral und c anschließendes Einbringen des Marknagels mit Schenkelhalsfraktur zum Retinieren der anatomischen Frakturreposition ausgetauscht oder durch eine Endoprothese ersetzt werden muss. Durch die Applikation von Zement oder dem zweioder mehrzeitigen Wechsel über einen Spacer können auf das vorliegende Keimspektrum abgeglichene Antibiotika lokal appliziert werden. Während Frühinfektionen mit nicht oder gering resistenten Keimlagen häufig mit einem ausgiebigen Débridement mit mechanischer Reinigung und anschließender antibiotischer Therapie unter Belassen des Osteosyntheseimplantats behandelt werden können, zeigen Hochresistenzerreger meist einen komplizierteren Verlauf. Diese bedingen in der Regel ein Austauschen des Implantats bzw. ein mehrzeitiges Vorgehen. Sollte es im Verlauf bereits zu einer ausreichenden knöchernen Konsolidierung gekommen sein, ist eine komplette Materialentfernung die Option der Wahl. Insbesondere bei älteren Patienten ist die Versorgung periimplantärer Infektionen, die sich häufig auf mehreren Etagen abbilden, problematisch und erfordert eine individuelle Strategie. Der Konzeptionierung der Wechseloperation (einzeitig, zweizeitig, mehrzeitig) kommt dabei eine entscheidende Bedeutung zu (- Abb. 4).

\section{Rotationsfehlstellung}

Auch Rotationsfehlstellungen zählen zu den häufigeren posttraumatischen Korrekturen. Die richtige Einstellung der Rotation im Bereich des Femurs sollte bereits intraoperativ erfolgen. Sollte eine Rotationsfehlstellung in einer frühen intraoperativen Revision nicht erkannt bzw. behoben werden können, so ist eine Revision im Verlauf im Sinne einer Rotationsosteotomie am proximalen Femur möglich. Hierzu sollte präoperativ die genaue Evaluation der Rotationsfehlstellung mithilfe einer Computertomographie (CT) zur Bestimmung der Antetorsion durchgeführt werden.

\section{Revision mittels Endoprothese}

Die endoprothetische Versorgung als Revisionsoperation nach fehlgeschlagener Osteosynthese zeigt eine gute
Ergebnisqualität [15]. Im Vergleich zu den Versorgungsergebnissen der Revisionsendoprothetik bei medialen Schenkelhalsfrakturen zeigt die Revisionsendoprothetik bei fehlgeschlagenen per-, inter- und subtrochantären Osteosynthesen deutlich schlechtere Ergebnisse $[1,15]$. Die aktuelle Literatur berichtet von höheren Revisions- und Komplikationsraten intraoperativ, schwieriger Implantatpositionierung sowie erhöhter postoperativer periprothetischer Fraktur- und Luxationsgefahr [1, 15]. Der entscheidende Muskel des Hüftgelenkes ist der am Trochanter-major-Massiv ansetzende Glutaeus medius. Bei nicht verheilten per- und intertrochantären Frakturen ist dieser häufig vom metaphysären Block gelöst. Die sichere Refixation mittels Drahtcerclagen oder Schraubenosteosynthese ist die Grundlage für ein ausreichend gutes Gangbild postoperativ. 

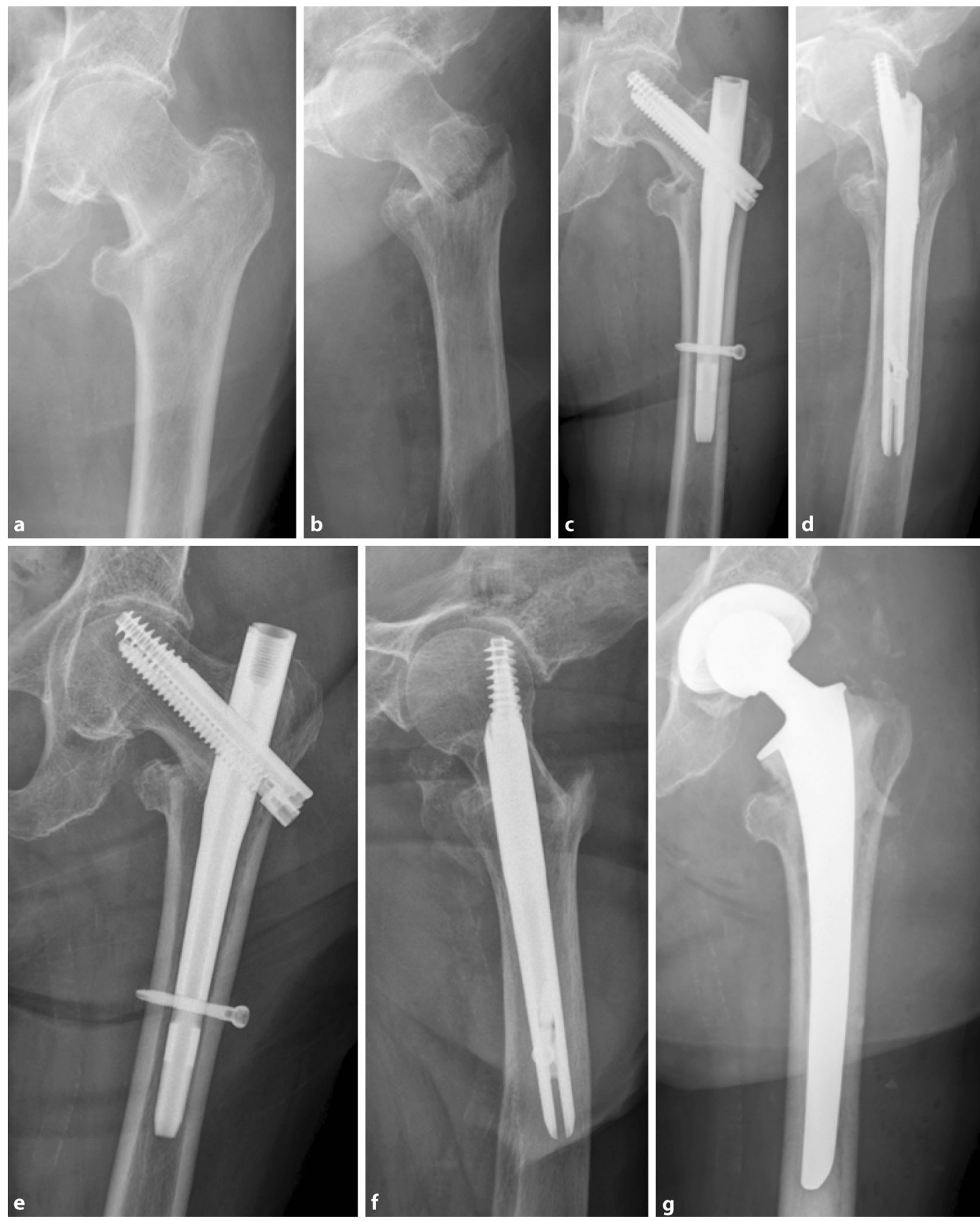

Abb. $3<$ a, b Pertrochantäre Femurfraktur AO-Typ 31-A1.2. c, d Postoperative Kontrolle, keine Center-Center-Position der Schenkelhalsschraube mit nichtoptimalem Tip-ToApex-Abstand. e, fCut-out der Schenkelhalsschraube im Verlauf. g Endoprothetischer Ersatz mittels Hybridhüfttotalendoprothese

\section{Primäre Hüftgelenkendo- prothetik bei per-, inter- und subtrochantären Frakturen}

Die Literaturlage ist recht eindeutig, dass die primäre Endoprothetik im Bereich von per-, inter- und subtrochantären Frakturen zu einer schlechteren Ergebnisqualität, zu einem höheren Blutverlust und zu einer erhöhten Mortalitätsrate im Vergleich zur osteosynthetischen Versorgung führt [5]. Dennoch kann die endoprothetische Versorgung in
Einzelfällen in der primären Situation indiziert sein [7]. Hierzu zählen schwere koxarthrotische Veränderungen im Bereich des Hüftgelenks. Aufgrund der Ankylose und der daraus resultierenden Steifigkeit im Hüftgelenk kommt es zu einer übermäßigen Bewegungseinleitung in die Fraktur und damit zu einer übermäßigen Beanspruchung des Osteosyntheseimplantats, was im Verlauf zu einem mechanischen Materialversagen führen kann.

\section{Fazit für die Praxis}

- Posttraumatische Korrekturen sind die Reserveoption bei fehlgeschlagenen Osteosynthesen.

- Da die Ergebnisqualität bei Revisionsoperationen in der Regel schlechter als bei adäquater Frakturheilung ist, kommt der Prävention eines Osteosyntheseversagens durch die Wahl des richtigen Implantats und der richtigen Technik eine entscheidende Bedeutung zu. 

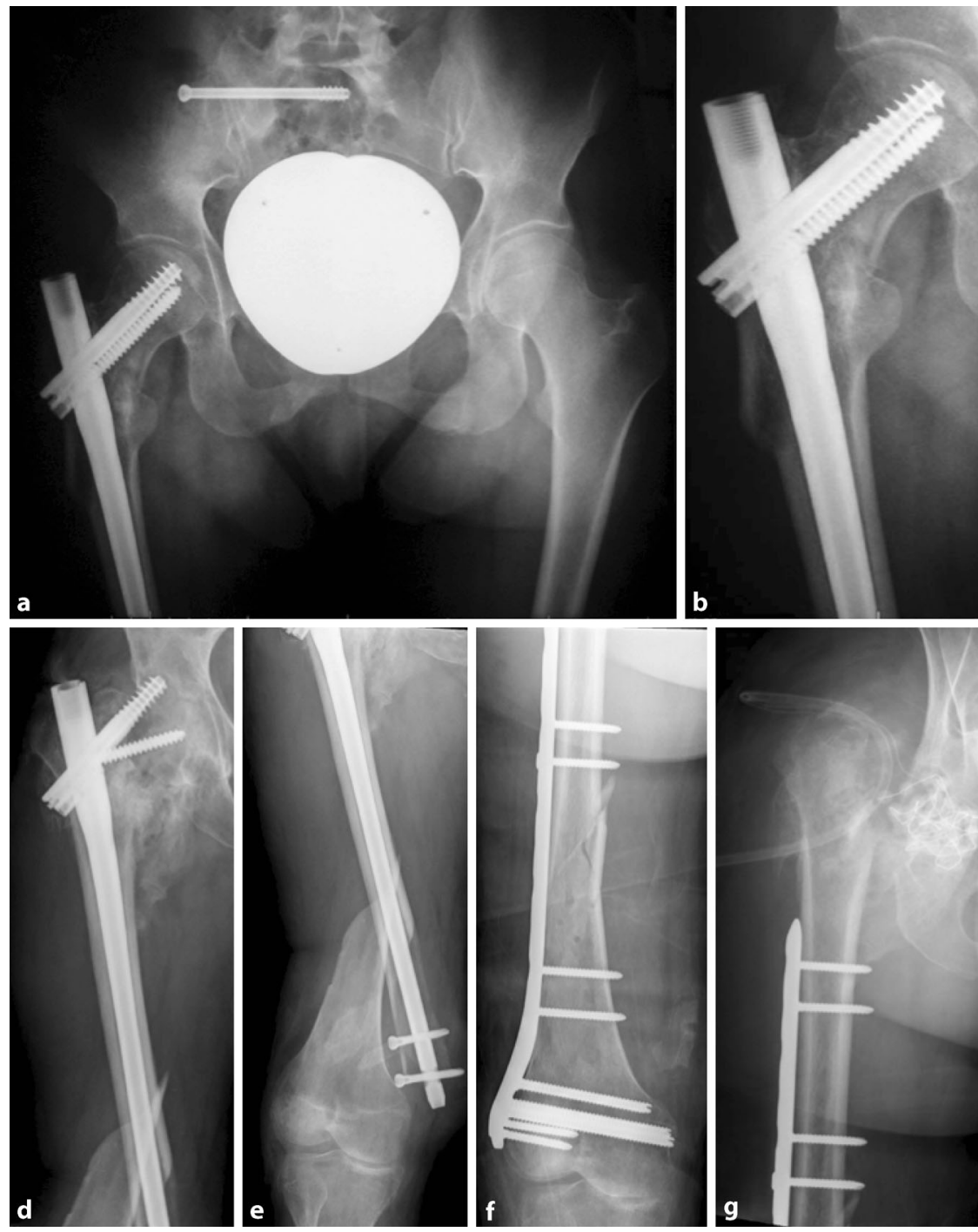

- Die frühzeitige Identifikation möglicher komplikationsträchtiger Verläufe ist von entscheidender Bedeutung.

- Insbesondere bei jungen Patienten steht ein biologischer Rekonstruktionsversuch im Vordergrund. Diese sind in der Regel erfolgreicher, je technisch inadäquater der Voreingriff war, je besser das proximale Kopffragment und die Gelenkqualität sind und je kopfferner das Versagen der Osteosynthese lokalisiert ist.

- Bei älteren Patienten ist in der Regel ein periprothetischer Ersatz notwendig.

- Bei allen Revisionen ist an mögliche Infektionen zu denken und das Vorliegen dieser mittels Punktion oder offener Biopsie zu sichern oder auszuschließen.

\section{Korrespondenzadresse}

\section{PD Dr. M. Hoffmann}

Klinik für Orthopädie und Unfallchirurgie,

Am Kiebitzberg 10, 23730 Neustadt in Holstein, Deutschland

MiHoffmann@schoen-kliniken.de

\section{Einhaltung ethischer Richtlinien}

Interessenkonflikt. M. Hoffmann, M. Runge, P. Dufek und K.-C. Westphal geben an, dass kein Interessenkonflikt besteht.

Dieser Beitrag beinhaltet keine von den Autoren durchgeführten Studien an Menschen oder Tieren.

The supplement containing this article is not sponsored by industry. Schön Klinik Neustadt
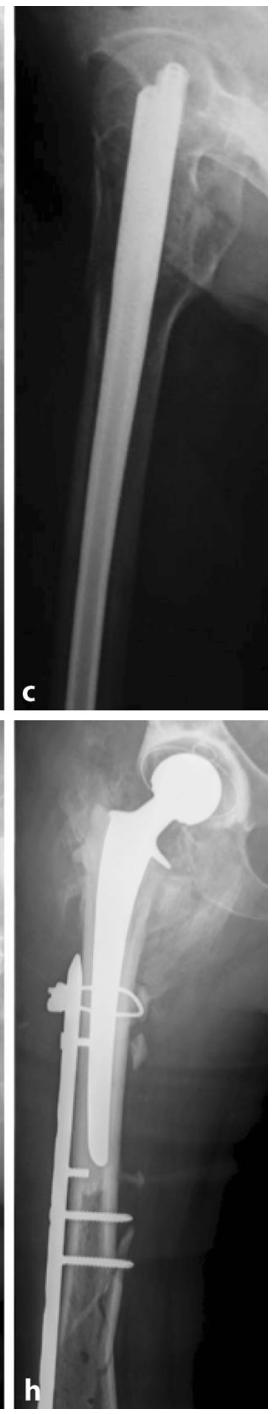

Abb. $4<$ a-c Postoperative Kontrolle nach Marknagelosteosynthese einer intertrochantären Femurfraktur. d, e Atraumatische, zweietagige Femurfraktur mit heterotopen Kalzifikationen als sekundäre Infektzeichen. f, $\mathbf{g}$, h Zweizeitiges Wechselkonzept mit Entfernung des Marknagels, Débridement, Plattenosteosynthese der distalen Femurfraktur. Spacer-Implantation und dann im Verlauf Implantation mit einer zementierten Hüftgelenktotalendoprothese

\section{Literatur}

1. Archibeck MJ, Carothers JT, Tripuraneni KR, White RE Jr. (2013) Total hip arthroplasty after failed internal fixation of proximal femoral fractures. J Arthroplasty 28(1):168-171. doi:10.1016/j.arth.2012.04.003.

2. Asayama I, Chamnongkich S, Simpson KJ, Kinsey TL, Mahoney OM (2005) Reconstructed hip joint position and abductor muscle strength after total hip arthroplasty. J Arthroplasty 20(4):414-420

3. Cruz-Sánchez M, Torres-Claramunt R, AlierFabregó A, Martínez-Díaz S (2015) Salvage for nail breakage in femoral intramedullary nailing. Injury 46(4):729-733. doi:10.1016/j.injury.2014.12.003.

4. Deakin DE, Guy P, O'Brien PJ, BlachutPA, Lefaivre KA (2015) Managing failed fixation: valgus osteotomy for femoral neck nonunion. Injury 46(3):492-496. doi:10.1016/j.injury.2014.11.022.

5. Dobbs RE, Parvizi J, Lewallen DG (2005) Perioperative morbidity and 30-day mortality after intertrochanteric hip fractures treated by internal fixation or arthroplasty. J Arthroplasty 20(8):963-966

6. Eschler A, Brandt S, Gierer P, Mittlmeier T, Gradl G (2014) Angular stable multiple screw fixation (Targon FN) versus standard SHS for the fixation of 
femoral neckfractures. Injury45(Suppl 1):S76-S80. doi:10.1016/j.injury.2013.10.026.

7. Hoffmann M, Hartel M, Rueger JM, Lehmann W (2014) Primary prosthetic replacement in per-and intertrochanteric fractures. Eur J Trauma Emerg Surg 40(3):273-277. doi:10.1007/s00068-0140412-9.

8. Inngul C, Blomfeldt R, Ponzer S, Enocson A (2015) Cemented versus uncemented arthroplasty in patients with a displaced fracture of the femoral neck: a randomised controlled trial. Bone Joint J 97-B(11):1475-1480. doi:10.1302/0301620X.97B11.36248

9. Liu P, WuX, Shi H, Liu R, Shu H, Gong J, Yang Y, Sun $Q$ Wu J, Nie X, Cai M (2015) Intramedullary versus extramedullary fixation in the management of subtrochanteric femur fractures: a meta-analysis. Clin Interv Aging 10:803-811 (eCollection 2015). doi:10.2147/CIA.S82119.

10. Parker MJ, Stedtfeld HW (2010) Internal fixation of intracapsular hip fractures with a dynamic locking plate: initial experience and results for 83 patients treated with a new implant. Injury 41(4):348-351. doi:10.1016/j.injury.2009.09.004.

11. Saß M, MittImeier T (2016) Joint-preserving treatment of medial femoral neck fractures with an angular stable implant. Oper Orthop Traumatol : (Epubahead of print)

12. Sakagoshi $D$, Sawaguchi $T$, Shima $Y$, Inoue D, Oshima T, Goldhahn S (2016) A refined definition improves the measurement reliability of the tip-apex distance. J Orthop Sci. doi:10.1016/j.jos.2016.03.001.

13. Thein R, Herman A, Kedem P, Chechik A, Shazar N (2014) Osteosynthesis of unstable intracapsular femoral neck fracture by dynamic locking plate or screw fixation: early results. J Orthop Trauma 28(2):70-76. doi:10.1097/BOT.0b013e3182a225

14. Trampuz A, Zimmerli W (2006) Diagnosis and treatment of infections associated with fracturefixation devices. Injury 37(Suppl 2):S59-S66

15. Zhang B, Chiu KY, Wang M (2004) Hip arthroplasty for failed internal fixation of intertrochanteric fractures. J Arthroplasty 19(3):329-333 